\title{
Presencia, severidad y frecuencia de síntomas en mujeres climatéricas pacientes del Instituto Nacional de Perinatología
}

\author{
Presence, severity and frequency of symptoms \\ in post-menopause women patients \\ of the National Institute of Perinatology
}

\author{
Evangelina Aldana Calva y Cecilia Mota González ${ }^{l}$
}

Citación: Aldana C., E. y Mota G., C. (2020). Presencia, severidad y frecuencia de síntomas en mujeres climatéricas pacientes del Instituto Nacional de Perinatología. Psicología y Salud, 30(2), 207-216. https://doi.org/10.25009/pys.v30i2.2655.

RESUMEN

El climaterio es una etapa complicada en sí misma: por un lado, por la connotación psicosocial que tiene, y por otro, por el momento en la vida en que se evidencian o exacerban diversos síntomas, algunos de los cuales tienen su origen en esta etapa de vida, y otros que siempre acompañan a la mujer. El propósito de este estudio fue conocer la presencia, severidad y frecuencia de síntomas en una muestra de 257 mujeres en climaterio, pacientes del Instituto Nacional de Perinatología de la Ciudad de México. Los resultados revelan que de los doce síntomas más frecuentes, cinco corresponden al factor emocional: tristeza, depresión, apatía, irritabilidad y cansancio; seis al factor de síntomas asociados a la vejez: dolor de huesos, dolor de espalda, dolor muscular, dolor articular, piel seca y problemas gastrointestinales, y solamente uno al factor de declive hormonal: el insomnio. Se concluye que los síntomas en el climaterio deben explicarse en términos más allá del mero declive hormonal.

Palabras clave: Climaterio; Síntomas; Frecuencia; Severidad.

\begin{abstract}
Menopause is a complicated stage in itself because of psychosocial connotations on the one hand, and because it occurs at a moment in life associated with symptoms that get evidenced or exacerbated on the other. Some such conditions start during this stage, and others have been accompanying women practically always. The purpose of the present study was to identify the presence, severity and frequency of symptoms in a sample of 257 post-menopausal patients of Mexico's Institute of Perinatology. Results revealed that, of the 12 most frequent symptoms, five correspond to emotional factors: sadness, depression, apathy, irritability and fatigue, and six to symptoms associated with old age: "bone pains", back pain, muscle pain, joint pain, dry skin and gastrointestinal problems. Only one symptom belonged to well documented hormonal decline factors: insomnia. It is concluded that post-menopausal symptoms must include explanations beyond hormonal decline.
\end{abstract}

Keywords: Climacteric; Symptoms; Frequency; Severity.

\footnotetext{
${ }^{1}$ Instituto Nacional de Perinatología, Coordinación de Psicología y Calidad, Montes Urales 800, Col. Lomas Virreyes, Miguel Hidalgo, 11000 Ciudad de México, México, tel. (55) 55-20-99-00, ext. 147, correo electrónico: alceva1964@yahoo.com.mx. Artículo recibido el 21 de agosto y aceptado el 14 de noviembre de 2019.
} 


\section{INTRODUCCIÓN}

$\mathrm{L}$ a esperanza de vida ha aumentado en los últimos años, ocasionando que la pirámide poblacional se haya invertido gracias a los avances en la farmacología, provocando que sean más las mujeres que se encuentran en la etapa de climaterio, ese periodo amplio de vida que abarca aproximadamente veinte años y que va de los 45 a los 65. Esta etapa se caracteriza por la presencia de cambios fisiológicos (síntomas vasomotores), biológicos (tránsito de la fase reproductiva a la no reproductiva), corporales (arrugas, encanecimiento o aumento de peso, entre otros), sociales (connotación psicosocial de esta etapa) y psicológicos (estado de ánimo), entre otros. A su vez, dichos cambios generan, evidencian o exacerban diversos síntomas que hacen que las mujeres busquen atención médica y, en muchas ocasiones, sean "medicalizadas". Si bien es cierto que esos indicios no siempre son de vida o muerte, se traducen en una disminución importante de la satisfacción en la mujer, lo que afecta distintas áreas de su vida (Aldana, Gómez, Sánchez y Gaviño, 2007).

Algunas de las situaciones asociadas al reporte de síntomas son las siguientes: a) el hecho de que la mujer se convierte en cuidadora de personas mayores, como los padres, y menores como los nietos; $b$ ) laboralmente, la mujer debe afrontar la jubilación, la posibilidad de carecer de empleo, o bien una sobrecarga de trabajo tanto físico como mental que favorece la aparición de procesos psicosociales y emocionales que deterioran la salud; c) la pérdida de la belleza y la juventud, sobrevaloradas en especial en la cultura latina; $d$ ) la pérdida de la capacidad reproductiva; $e$ ) el reencuentro con la pareja; $f$ ) en algunos casos, el surgimiento del síndrome del llamado "nido vacío"; $g$ ) el comienzo de los padecimientos propios de la vejez; h) una historia de vida no resuelta, lo que favorece que se conjuguen el pasado y el presente, convirtiéndose en un detonador del malestar emocional.

Todo lo anterior ha llevado a los especialistas a buscar y profundizar en algunos factores que puedan ayudar a la comprensión del comportamiento y la actitud de la mujer en esta etapa de vida (Aldana et al., 2007; Couto y Nápoles, 2014).

El climaterio es una experiencia individual en la que la sintomatología depende de la fuerza, la vitalidad y el conocimiento que se tenga acerca de esta etapa, la cual además está matizada por diversos factores culturales y educativos, diferentes en cada grupo social. Es importante señalar que cada mujer vive de forma diferente la aparición, intensidad, duración, signos y síntomas de dicha etapa, y que hay muchas mujeres que no atraviesan por dicha experiencia (Carrobles, Velasco y Gómez, 2015).

En las siguientes líneas se describen algunos hallazgos sobre este tópico. Así, Pérez, Martínez, López y Ornat (2013) señalan que los síntomas más frecuentes encontrados en su investigación fueron los músculo-esqueléticos, los sofocos y la sudoración, y que los síntomas psicológicos y urogenitales tienen una relación directa con la edad, la masa corporal, la edad al ocurrir la menopausia y la edad de la pareja sexual.

En 2016, Martínez, Sarduy, Rodríguez, Rodríguez e Iglesias encontraron que una cuarta parte de las mujeres de su muestra no manifestaban síntomas, y que del 75 por ciento restante $37.4 \%$ los percibía como leves, en tanto que $37.6 \%$ los describió como moderados o severos. Los autores concluyen que un estado de vida saludable funciona como una protección contra la aparición de síntomas.

Carvajal, Flores, Marín y Morales (2016) señalan la escasa investigación realizada en este sector de la población respecto a la ansiedad; sin embargo, en el área clínica se sabe que aquella aumenta durante la menopausia.

A su vez, Jaramillo, Martínez y Jaramillo (2016) apuntan que la menopausia y el desajuste hormonal propio de esta etapa generan cambios que van de leves a severos, los que en ocasiones interfieren considerablemente en la vida cotidiana de la mujer; tal es el caso de la osteoporosis, el aumento del riesgo cardiovascular o la resistencia a la insulina, entre muchos otros.

Heras et al. (2017) llevaron a cabo un estudio en el cual las mujeres señalaron que los cambios en su humor se asociaban con no estar preparadas para esta etapa, además de que no conocían con precisión cuándo se presentaba.

Charabati, Sanabria y Sánchez (2017) indican que los síntomas vasomotores son el sello distintivo del climaterio y que los experimenta una gran parte de las mujeres en etapa postmenopáu- 
sica. Las mujeres con obesidad o sobrepeso refirieron con más frecuencia los bochornos, el cual es uno de los síntomas más característicos, por lo que buscaban atención médica en virtud de que alteraban su descanso nocturno.

Mercado, Monterrosa y Durán (2018), por su parte, señalan la etnia como un factor principal para la aparición de síntomas, ya que este factor determina el funcionamiento del eje hipotálamohipófisis-ovarios, las concentraciones séricas de estradiol, testosterona y globulina fijadora de hormonas sexuales, encontrando diferencias significativas entre mujeres colombianas, españolas y afrodescendientes.

Romero, Monterrosa y Paternina (2010) llevaron a cabo una revisión de la literatura especializada, hallando que $78 \%$ de los diecinueve estudios revisados mostraban diferencias significativas respecto a la presencia de síntomas, las que atribuyeron a la etnia de pertenencia.

Escobar, Chica y Cuevas (2008) refieren en su estudio que los trastornos del sueño son patologías cada vez más frecuentes relacionadas con los cambios hormonales, por lo que las mujeres en climaterio los sufren de manera habitual, llegando en algunas ocasiones a modificar su estilo de vida, lo que coincide con lo ya planteado por Bélisle et al. (2006), quienes refieren que dos de los principales trastornos del sueño son la falta de éste o la dificultad para conciliarlo, los que aparecen en la perimenopausia y la menopausia. En algunos casos esos trastornos se relacionan con problemas vasomotores, como bochornos y sudoraciones, lo que se conoce como "efecto dominó". Tales problemas interfieren de forma importante en la calidad de vida de las mujeres.

Respecto a los síntomas relacionados a la sexualidad, Lugones (2013) encuentra que la misma, además de estar regulada por factores biológicos, también está a expensas de los factores sociales, culturales y económicos. Las condiciones de género afectan esta área de manera directa ya que las desigualdades desencadenan agotamiento mental y psicológico en las mujeres en esta etapa de la vida, de tal suerte que en ellas se modifica la respuesta sexual humana de manera negativa, y no es sino hasta que ocurre un reajuste hormonal y emocional que se vuelve a equilibrar, siempre y cuando antes de esa etapa no haya surgido algún trastorno o disfunción sexual.

La presencia de síntomas no solamente altera el estado de salud, sino también el entorno familiar, social, laboral y económico, es decir, la calidad de vida en general. En una muestra de 472 mujeres, Ayala, Muñoz, Celedonio, Rivera y Ayala (2003) encontraron que la calidad de vida disminuye a partir de la perimenopausia y continúa disminuyendo conforme pasa el tiempo, ocurriendo el deterioro más notorio en las áreas psicosocial y física. En tanto, Ulloque, Carrianzo, Monterrosa y Paternina (2013) observaron en una muestra de 760 mujeres que el $86 \%$ de ellas sufría oleadas de calor -manifestación comúnmente asociada a la menopausia-, siendo leves en la premenopausia y moderadas en la perimenopausia y posmenopausia, y que se acompañaban de dolores musculoarticulares, cansancio físico y mental, irritabilidad y problemas vesicales y sexuales. Por su parte, Blumem et al. (2011) hallaron que entre más intensos eran los síntomas vasomotores, más pobre era la calidad de vida, y que además esos síntomas aumentaban significativamente con la edad.

Debido a esa gama de síntomas, en décadas recientes se ha intentado clasificarlos; entre esos intentos se encuentran los de Utian (1980), quien los catalogó de acuerdo al órgano afectado; Lauritzen (1984) utiliza cada fase del climaterio; Comino y Gomiz (1991) señalan los síntomas en relación al climaterio natural o quirúrgico; Pacheco (1994) los clasifica en tempranos y tardíos; Morales, Díaz y Aldana (1995) encontraron que seis de los diez síntomas, reportados como más frecuentes, eran psicológicos; Martínez (2007) presenta una clasificación que considera las alteraciones en tres niveles: neurovegetativas, psicológicas y genitourinarias; Aldana et al. (2007) señalan que los síntomas asociados al climaterio son de orden fisiológico (vasomotores), ginecológico (incontinencia urinaria y sangrado vaginal), psicológico (estados de ánimo somáticos) y mixto (disfunción sexual). Asimismo, Couto y Nápoles (2014) plantean que los síntomas más frecuentes en la etapa climatérica son de origen psicológico, y aunque no generan problemas graves de salud mental, pueden disminuir la satisfacción personal e influir en las relaciones interpersonales y familiares, así como también en las sexuales, laborales y de pareja. 
El abordaje y la explicación de los síntomas en el climaterio han sido hechos a partir de diferentes perspectivas. Así, el modelo biomédico los explica a partir del declive hormonal; luego entonces, indica la ingesta de hormonas (Alvarado et al., 2015). El modelo psicológico opta por la historia de vida, el aprendizaje y demás, por lo que indica el tratamiento psicoterapéutico (Aldana et al., 2007). En el otro extremo, la medicina bioenergética explica al ser humano de manera holística y trata los síntomas mediante un conjunto de métodos no convencionales, como los medicamentos o remedios de origen animal, vegetal o mineral (García, Selva, Pérez y Pardo, 2012).

Por lo anterior, el presente estudio descriptivo, de diseño no experimental, transversal, con un muestreo no probabilístico e intencional por cuota, tuvo como objetivo conocer la presencia, severidad y frecuencia de la sintomatología que manifestaban mujeres en etapa del climaterio, pacientes del Instituto Nacional de Perinatología. Cabe señalar que se aplicó un instrumento diseñado específicamente para esta población, mismo que enlista los 34 síntomas encontrados en la literatura especializada y que detecta su ocurrencia y la severidad y frecuencia de cómo las citadas mujeres los percibían. Debe decirse que el trabajo forma parte de una investigación más amplia, en la que se analizan diversos datos sobre sexualidad, satisfacción sexual y marital, ansiedad y depresión, entre otros. En este estudio únicamente se reporta la ocurrencia, la frecuencia y la severidad de los síntomas.

\section{MÉTODO}

\section{Participantes}

De manera inicial, participaron en el estudio 259 mujeres, cuya edad fluctuó entre 37 y 61 años, con una media de 49.31 (D.E. = 5.352). Para el análisis solo se tomaron los datos de 257 participantes, ya que dos de ellas se eliminaron por no contestar el instrumento en su totalidad.

Los datos sociodemográficos muestran que en estas mujeres se mantenían algunos rasgos de la cultura tradicional, la que se distingue por los ro- les de género, lo que se observa en la escolaridad, la cual dio una media de 9.95 años. En cuanto a su estado civil, $79.5 \%$ eran casadas, $12 \%$ tenían pareja, aunque no vivían con ella, y $8.5 \%$ estaban en unión libre. En lo relativo a su ocupación, 59.5\% se dedicaba al hogar y $33.6 \%$ a trabajar fuera de este. Respecto a la etapa de vida, $29.7 \%$ se hallaba en la premenopausia, $19.3 \%$ en la menopausia y $50.2 \%$ en la posmenopausia. De las participantes, $17.2 \%$ utilizaba hormonales, porcentaje del cual ocho eran premenopáusicas, siete menopáusicas y nueve posmenopáusicas.

Los criterios de inclusión fueron recibir atención en la clínica de climaterio del Instituto Nacional de Perinatología; cursar un climaterio natural, precoz o quirúrgico, y contar con un nivel de estudios mínimos de educación primaria a fin de poder comprender los reactivos del instrumento.

El criterio de exclusión fue presentar cuadros psicopatológicos, con base en los criterios del DSM-IV, y el de eliminación no responder el instrumento en su totalidad.

\section{Instrumento}

Encuesta de síntomas (Morales et al., 1995).

Se aplicó la encuesta de síntomas, que contiene 34 reactivos los cuales miden la presencia, severidad y frecuencia de síntomas asociados al climaterio, y que se divide en dos apartados: datos sociodemográficos, y detección de la presencia, severidad y frecuencia de síntomas. Este instrumento fue construido intencionalmente para llevar a cabo estudios con mujeres en la etapa de climaterio.

La confiabilidad del instrumento tiene un coeficiente alfa de Cronbach de .93, con 31 síntomas. Para determinar la validez del instrumento se efectuó un análisis factorial exploratorio con rotación varimax, el cual explicó $49.6 \%$ de la varianza total.

\section{Procedimiento}

La aplicación se llevó a cabo en un cubículo expresamente conformado para la aplicación de instrumentos en la Torre de Investigación del Departamento de Psicología. Dicha aplicación se hizo en una sola sesión por personal capacitado para ello. 
Las pacientes fueron informadas del objetivo de la investigación, y pidiéndoles que, en caso de no estar de acuerdo, lo manifestaran libremente ya que eso no afectaría su atención en la institución. Se subrayó el carácter voluntario de su participación y se aseguró la confidencialidad de los datos. Todas ellas firmaron un formato de consentimiento informado.

\section{RESULTADOS}

En cuanto a la presencia de síntomas, se encontró que, de los doce más frecuentes, seis eran de tipo psicológico: cansancio, tristeza, irritabilidad, piel seca, depresión, dolor muscular, dolor articular, problemas gastrointestinales, dolor de espalda baja, dolor de huesos y apatía. Se incluye el insomnio ya que muestra un puntaje igual al de la apatía. Tales datos se muestran en la Tabla. 1.

Tabla 1. Presencia y frecuencia de síntomas.

\begin{tabular}{|l|c|c|c|c|}
\hline \multicolumn{1}{|c|}{ Síntoma } & $\begin{array}{c}\text { Pacientes } \\
\text { con síntoma }\end{array}$ & $\begin{array}{c}\text { F } \\
\text { \% }\end{array}$ & $\begin{array}{c}\text { Pacientes } \\
\text { sin síntoma }\end{array}$ & $\begin{array}{c}\text { F } \\
\text { \% }\end{array}$ \\
\hline Cansancio & 245 & 95.5 & 12 & 4.5 \\
\hline Tristeza & 242 & 94.1 & 15 & 5.8 \\
\hline Irritabilidad & 230 & 89.4 & 27 & 10.5 \\
\hline Piel seca & 218 & 84.8 & 39 & 15.1 \\
\hline Depresión & 217 & 84.4 & 40 & 15.5 \\
\hline Dolor muscular & 213 & 82.2 & 44 & 17.1 \\
\hline Dolor articular & 213 & 82.2 & 44 & 17.1 \\
\hline Trastornos gastrointestinales & 213 & 82.2 & 44 & 17.1 \\
\hline Dolor de espalda baja & 204 & 79.3 & 53 & 20.6 \\
\hline Dolor de huesos & 200 & 77.8 & 57 & 22.1 \\
\hline Apatía & 199 & 77.4 & 58 & 22.5 \\
\hline Insomnio & 199 & 77.4 & 58 & 22.5 \\
\hline
\end{tabular}

En cuanto a la severidad y frecuencia de esos seis síntomas, en la Tabla 2 se puede observar que, de las mujeres que refieren los síntomas psicológicos antedichos, el mayor porcentaje los padecía de manera severa y frecuente, mientras que, respecto de la apatía, la mayor parte de las pacientes que la sufría la consideró como leve y con escasa frecuencia.

Tabla 2. Presencia, severidad y frecuencia de síntomas.

\begin{tabular}{|c|c|c|c|c|c|c|c|c|c|c|c|c|}
\hline Síntomas & \multicolumn{2}{|c|}{ Cansancio } & \multicolumn{2}{|c|}{ Tristeza } & \multicolumn{2}{|c|}{ Irritabilidad } & \multicolumn{2}{|c|}{ Piel seca } & \multicolumn{2}{|c|}{ Depresión } & \multicolumn{2}{|c|}{$\begin{array}{c}\text { Dolor } \\
\text { muscular }\end{array}$} \\
\hline Sin síntomas & \multicolumn{2}{|c|}{12} & \multicolumn{2}{|c|}{15} & \multicolumn{2}{|c|}{27} & \multicolumn{2}{|c|}{39} & \multicolumn{2}{|c|}{40} & \multicolumn{2}{|c|}{44} \\
\hline $\begin{array}{l}\text { Total } \\
\text { de pacientes }\end{array}$ & \multicolumn{2}{|c|}{245} & \multicolumn{2}{|c|}{242} & \multicolumn{2}{|c|}{230} & \multicolumn{2}{|c|}{218} & \multicolumn{2}{|c|}{217} & \multicolumn{2}{|c|}{213} \\
\hline \multirow{3}{*}{ Leves } & $32 *$ & \multirow{3}{*}{55} & 32 & \multirow{3}{*}{71} & 29 & \multirow{3}{*}{68} & 36 & \multirow{3}{*}{76} & 40 & \multirow{3}{*}{65} & 29 & \multirow[t]{3}{*}{57} \\
\hline & $13 * *$ & & 16 & & 29 & & 24 & & 6 & & 19 & \\
\hline & $10 * * *$ & & 23 & & 10 & & 16 & & 19 & & 9 & \\
\hline \multirow{3}{*}{ Moderados } & $9 *$ & \multirow{3}{*}{89} & 12 & \multirow{3}{*}{78} & 6 & \multirow{3}{*}{73} & 10 & \multirow{3}{*}{70} & 7 & \multirow{3}{*}{61} & 7 & \multirow[t]{3}{*}{70} \\
\hline & $41 * *$ & & 29 & & 31 & & 30 & & 27 & & 24 & \\
\hline & $39 * * *$ & & 37 & & 36 & & 30 & & 27 & & 39 & \\
\hline \multirow{3}{*}{ Severos } & $20^{*}$ & \multirow{3}{*}{101} & 16 & \multirow{3}{*}{93} & 18 & \multirow{3}{*}{89} & 8 & \multirow{3}{*}{72} & 17 & & 15 & 86 \\
\hline & $29 * *$ & & 17 & & 20 & & 10 & & 18 & 91 & 12 & \\
\hline & $52 * * *$ & & 60 & & 51 & & 54 & & 56 & & 59 & \\
\hline
\end{tabular}

*Pocas veces, **Algunas veces, ***Frecuentemente. 
Tabla 2 (Continúa)

\begin{tabular}{|c|c|c|c|c|c|c|c|c|c|c|c|c|}
\hline Síntomas & \multicolumn{2}{|c|}{$\begin{array}{c}\text { Problemas } \\
\text { gastrointestinales }\end{array}$} & \multicolumn{2}{|c|}{$\begin{array}{c}\text { Dolor } \\
\text { Articular }\end{array}$} & \multicolumn{2}{|c|}{$\begin{array}{c}\text { Dolor } \\
\text { de espalda }\end{array}$} & \multicolumn{2}{|c|}{$\begin{array}{c}\text { Dolor } \\
\text { de huesos }\end{array}$} & \multicolumn{2}{|c|}{ Apatía } & \multicolumn{2}{|c|}{ Insomnio } \\
\hline Sin síntomas & \multicolumn{2}{|c|}{44} & \multicolumn{2}{|c|}{44} & \multicolumn{2}{|c|}{53} & \multicolumn{2}{|c|}{57} & \multicolumn{2}{|c|}{58} & \multicolumn{2}{|c|}{58} \\
\hline $\begin{array}{l}\text { Total } \\
\text { de pacientes }\end{array}$ & \multicolumn{2}{|c|}{213} & \multicolumn{2}{|c|}{213} & \multicolumn{2}{|c|}{204} & \multicolumn{2}{|c|}{200} & \multicolumn{2}{|c|}{199} & \multicolumn{2}{|c|}{199} \\
\hline \multirow{3}{*}{ Leves } & $37 *$ & \multirow{3}{*}{66} & 43 & \multirow{3}{*}{82} & 26 & \multirow{3}{*}{72} & 19 & \multirow{3}{*}{50} & 49 & \multirow{3}{*}{81} & 33 & \multirow{3}{*}{66} \\
\hline & $13 * *$ & & 27 & & 32 & & 18 & & 16 & & 18 & \\
\hline & $16 * * *$ & & 12 & & 14 & & 13 & & 16 & & 15 & \\
\hline \multirow{3}{*}{ Moderados } & 9* & \multirow{3}{*}{79} & 13 & \multirow{3}{*}{82} & 7 & \multirow{3}{*}{71} & 10 & \multirow{3}{*}{73} & 13 & \multirow{3}{*}{53} & 11 & \multirow{3}{*}{64} \\
\hline & $35 * *$ & & 25 & & 29 & & 34 & & 21 & & 25 & \\
\hline & $35^{* * *}$ & & 44 & & 35 & & 29 & & 19 & & 28 & \\
\hline \multirow{3}{*}{ Severos } & $11 *$ & \multirow{3}{*}{68} & 6 & \multirow{3}{*}{49} & 3 & \multirow{3}{*}{61} & 11 & \multirow{3}{*}{77} & 12 & & 13 & \\
\hline & $5 * *$ & & 15 & & 7 & & 26 & & 15 & 65 & 7 & 69 \\
\hline & $52 * * *$ & & 28 & & 51 & & 40 & & 38 & & 49 & \\
\hline
\end{tabular}

*Pocas veces, **Algunas veces, ***Frecuentemente

En lo que se refiere al conocimiento y la información acerca del climaterio, así como el comportamiento de los síntomas en los tres factores que comprende el instrumento, se encontró que el factor 1 se compuso de nueve síntomas, al igual que el factor 3, mientras que el factor 2 incluyó diez. En la Tabla 3 se describen dichos resultados.

Tabla 3. Síntomas que componen los factores del instrumento y porcentaje de pacientes que los presentan.

\begin{tabular}{|c|c|c|c|c|c|}
\hline \multicolumn{2}{|c|}{$\begin{array}{c}\text { Factor } 1 \\
\text { Síntomas emocionales } \\
(\%)\end{array}$} & \multicolumn{2}{|c|}{$\begin{array}{c}\text { Factor } 2 \\
\text { Síntomas asociados } \\
\text { a la vejez o desgaste del organismo } \\
(\%) \\
\end{array}$} & \multicolumn{2}{|c|}{$\begin{array}{c}\text { Factor } 3 \\
\text { Síntomas asociados } \\
\text { al declive hormonal } \\
(\%)\end{array}$} \\
\hline Cansancio & 95.3 & Piel seca & 84.8 & Insomnio & 77.4 \\
\hline Tristeza & 94.1 & Problemas gastrointestinales & 82.8 & Cefaleas & 76.6 \\
\hline Irritabilidad & 89.4 & Dolor muscular & 82.8 & Zumbido de oídos & 75.0 \\
\hline Depresión & 84.4 & Dolor articular & 82.8 & Bochornos & 70.4 \\
\hline Apatía & 77.4 & Dolor de espalda & 79.3 & Ansiedad & 70.4 \\
\hline Llanto & 76.2 & Dolor de huesos & 77.8 & Sudoración nocturna & 69.2 \\
\hline Labilidad emocional & 73.9 & Sequedad vaginal & 74.2 & Calambres & 65.7 \\
\hline Minusvalía & 65.3 & Parestesias & 72.2 & Prurito vulvar & 38.9 \\
\hline Desadaptación & 63.7 & Incontinencia & 53.2 & Lipotimias & 21.7 \\
\hline & & Dispareunia & 45.9 & & \\
\hline
\end{tabular}

\section{DISCUSIÓN}

Antes de indicar tratamientos médicos, psicológicos o psiquiátricos a las mujeres en etapa de climaterio, es fundamental explorar tres puntos que darán pie a tales tratamientos: 1) identificar si el origen de los síntomas es el declive hormonal, o si este solamente los exacerba; 2) averiguar si los síntomas de tipo psicológico han acompañado a la mujer a lo largo de su vida, o bien se han generado en esta etapa particular, y 3) detectar los síntomas que son adaptativos y los que constituyen focos de alarma que tienen una connotación clínica importante (Aldana et al., 2007).

Los resultados de la presente investigación hacen posible afirmar que las mujeres de la muestra estudiada, en gran medida, continuaban manteniendo patrones de conducta características del 
tipo de crianza de décadas anteriores; sin embargo, hubo algunos casos en los que contaban con pareja, pero sin vivir juntos, lo que da pie a pensar que hay una mayor aceptación de otras formas de relacionarse con la pareja. Referente a su ocupación, un porcentaje considerable de las participantes se hallaban insertas en el área laboral como parte de la fuerza productiva, independientemente del tipo de trabajo que llevaran a cabo. En cuanto a la etapa de vida, la mitad de la muestra se encontraba en la fase posmenopáusica, en tanto que, respecto a la edad, casi la mitad de ellas rebasaba los cincuenta años, lo que posiblemente se relacione con los doce síntomas más frecuentes; de hecho, la piel seca, el dolor muscular, articular y de huesos, así como los problemas gastrointestinales, son propios del inicio de la vejez.

Se observó que la población estudiada era decididamente sintomática, toda vez que, de la muestra de 257 mujeres, todas ellas padecía al menos un síntoma, lo que concuerda con estudios tales como el de Dorador y Orozco (2018), quienes encontraron que la totalidad de su muestra mostraba al menos un síntoma; Hernández et al. (2010), que señalan que $66.8 \%$ de las mujeres de su estudio estaba en esa misma condición; Martínez et al. (2016), que reportan que la proporción era de tres cuartas partes de su muestra, y Contreras y González (2015), quienes puntualizan que casi noventa por ciento de su muestra exhibía asimismo al menos uno de tales síntomas.

Morales et al. (1995) emplearon la misma encuesta de síntomas, hallando que, de los diez síntomas más frecuentes, seis implicaban aspectos psicológicos, como tristeza, irritabilidad, cansancio, depresión, insomnio y llanto, en tanto que los resultados de la presente investigación reflejan que, entre los doce más frecuentes, cinco eran de tipo psicológico: cansancio, tristeza, irritabilidad, depresión y apatía. Tales resultados coinciden con los de Legorreta, Montaño, Hernández, Salinas y Hernández (2013), quienes encontraron que entre los síntomas más frecuentes estaban el cansancio, la irritabilidad y la depresión. Otro estudio que concuerda con los resultados obtenidos aquí es el de Hernández et al. (2010), que señalan que entre los doce síntomas más frecuentes se encuentran la irritabilidad y el llanto fácil, ocupando la irritabilidad el tercer lugar.
Por lo anterior, puede decirse que, en efecto, los síntomas psicológicos constituyen una parte importante en la vida de las mujeres que se hallan en el climaterio, lo que amerita una atención particular en los estudios de los determinantes biológicos, sociales, culturales o económicos, entre otros, para así evitar sus efectos negativos en la calidad de vida.

A la encuesta de síntomas se le hizo un análisis factorial con rotación varimax para determinar los factores de que está compuesta, obteniéndose tres: 1 : síntomas emocionales, 2 : síntomas asociados a la vejez o desgaste del organismo, y 3: síntomas asociados al declive hormonal. Estos factores podrían servir de base a la propuesta de una nueva clasificación de la sintomatología en este sector de la población, producto de un trabajo más fino empleando el mismo instrumento que Morales et al. (1995), la que englobaría así las clasificaciones ya existentes de Utian (1980), Lauritzen (1984), Comino y Gomiz (1991), Morales et al. (1995), Martínez (2007) y Aldana et al. (2007), entre algunas otras.

En cuanto al comportamiento de la sintomatología, el instrumento empleado permite observar la distribución de los síntomas entre los tres factores que lo componen, encontrando que de los doce más frecuentes, cinco corresponden al factor emocional (tristeza, depresión, apatía, irritabilidad y cansancio), seis al factor de síntomas asociados a la vejez (dolor de huesos, dolor de espalda, dolor muscular, dolor articular, piel seca y problemas gastrointestinales), y solamente uno del factor correspondiente al declive hormonal (insomnio). Lo anterior coincide con lo planteado por Couto y Nápoles (2014), quienes señalan que los síntomas más frecuentes en esta etapa de vida son los psicológicos.

En el estudio ya referido de Morales et al. (1995), el insomnio formaba parte de los síntomas psicológicos, en tanto que en la presente investigación, como resultado de la rotación varimax, formó parte del factor 3 , que hace referencia a los síntomas asociados al declive hormonal, lo que coincide con lo planteado por Bélisle et al. (2006) y Escobar et al. (2008), quienes señalan que los trastornos del sueño están asociados al declive hormonal, que aquí ocupó el doceavo lugar. Es importante estudiar dicho síntoma ya que se sabe 
que tales trastornos favorecen el desarrollo de enfermedades crónico-degenerativas y que alteran la calidad de vida, según refieren Lopera, Parada, Martínez, Jaramillo y Rojas (2016). Además, es necesario saber si hay obesidad y si esta se relaciona con los referidos trastornos del sueño, en particular con el insomnio, tal como lo indican Charabati et al. (2017).

En cuanto a los síntomas psicológicos, valdría la pena llevar a cabo investigaciones más profundas en las que se indague su origen, ocurrencia y severidad. El resultado obtenido correspondiente al área psicológica fortalece la necesidad de brindar la atención a este sector de la población ya que, como indican Couto y Nápoles (2014), los síntomas psicológicos no generan problemas graves de salud mental, pero pueden disminuir la satisfacción personal e influir negativamente en las relaciones interpersonales, familiares, de pareja, sexuales y laborales.

En cuanto a la severidad se encontró que absolutamente todos los síntomas iban de leves a severos; de hecho, cuatro de los cinco síntomas psicológicos fueron referidos como severos; solo dos de los asociados a la vejez fueron considerados como tales, al igual que el único asociado al declive hormonal, que es el insomnio Estos resultados concuerdan con lo señalado por Lopera et al. (2016).

Es probable que en este estudio las mujeres hayan percibido cambios importantes en varias áreas de su vida y los hayan ignorado al carecer de los conocimientos o la información necesaria acerca de lo que implican la menopausia y el climaterio (43.3\% de ellas desconocían las implicaciones de esta etapa), lo que las lleva a desarrollar síntomas de tipo emocional; es sabido que el estado de ánimo está asociado al desconocimiento de lo que implica esta etapa, pues la mujer no está preparada para enfrentar las modificaciones corporales, fisiológicas y sociales que acompañan el climaterio. Sin duda, esto pone a prueba la estructura de personalidad y la fortaleza emocional de la mujer; si estas se encuentran fracturadas, la mujer vivirá graves complicaciones emocionales, según indican Aldana et al. (2007) y Heras et al. (2017).

Síntomas como el dolor de huesos, dolor de espalda, dolor muscular, dolor articular, piel seca y problemas gastrointestinales -síntomas todos ellos propios de vejez- se explican por la edad de las pacientes ya que más de la mitad se encontraban en la posmenopausia, la que ocurre, la mayoría de veces, después de los cincuenta años. Estos resultados difieren de los obtenidos por Hernández et al. (2010), quienes encontraron que los síntomas reportados con más frecuencia fueron los bochornos, la irritabilidad, el nerviosismo y los mareos.

La contribución de esta investigación radica en el concentrado de síntomas que muy probablemente puede sufrir la mujer que transita por el climaterio. La aplicación de instrumentos de este tipo, los cuales evalúan la severidad y la frecuencia de esos síntomas, así como la historia clínica, hace posible formular o plantear modelos de atención interdisciplinarios o especializados en las instituciones de salud.

El trabajo de los psicólogos respecto al climaterio en las instituciones de salud ocurre en varios niveles. Primeramente, en la psicoeducación, la cual está enfocada a informar sobre los cambios y síntomas que aparecen durante el climaterio, a fin de diluir o eliminar los prejuicios e ideas equívocas sobre esta etapa, trabajo que se lleva a cabo con el personal de los diversos servicios (trabajadores sociales, enfermeros, médicos, personal administrativo, etc.), con el público en general y en particular con las mujeres que atraviesan esta etapa. En segundo lugar, en la sensibilización del área médica, donde se hace hincapié en la importancia de una atención con calidad y calidez, para lo cual deben ser tomados en cuenta no solo los cambios hormonales, sino también los sociales, económicos y culturales de las mujeres. Por último, en la atención psicológica, en la que es esencial el diagnóstico y el modelo idóneo de tratamiento, para lo que se requieren instrumentos que detecten los síntomas y su frecuencia y severidad, más la elaboración de la historia clínica.

Los síntomas referidos por las mujeres deben ser tratados, sin importar su origen, ya que de no ser así sobreviene un malestar psicológico que, si bien es cierto no lleva a la mujer a la muerte, se traduce en una notable disminución de la satisfacción personal, lo que influye de manera negativa en las diferentes áreas de su vida. 


\section{REFERENCIAS}

Aldana C., E., Gómez L., M.E., Sánchez P., M.A., y Gaviño G., F. (2007). Áreas de conflicto en mujeres en la etapa del climaterio en dos grupos de psicoterapia. Ginecología y Obstetricia de México, 75, 332-340.

Alvarado G., A., Hernández Q., T., Hernández V., M., Negrín P., M.C., Ríos C., B., Valencia P., G.U., Vital R., V.S., Basavilvazo R., M.A., Torres A., L.P., Ortiz L., G.F., Sánchez A., F. y Montaño U., A. (2015). Guía de práctica clínica. Diagnóstico y tratamiento de la perimenopausia y la posmenopausia. Revista Médica del Instituto Mexicano del Seguro Social, 53(2), 214-225.

Ayala, F., Muñoz, R., Celedonio, M., Rivera, J. y Ayala R. (2003). Calidad de vida en el climaterio. Revista Peruana de Ginecología y Obstetricia, 49(3), 160-165.

Bélisle, S., Blake, J., Basson, R., Desindes, S., Graves, G., Grigoriadis, S., Johnston, S., Lalonde, A., Mills, C., Nash, L., Reid, R., Rowe, T., Senikas, V., Senikas, V. y Turek, M. (2006). Consensus Conference on Menopause. Journal of Obstetrics and Gynaecology Canada, 28(2), 57-94.

Blümel, J.E., Chedraui, P., Barón, G., Belzares, E., Bencosme, A., Calle, A., Danckers, L., Espinoza, M.T., Flores, D., Gómez, G., Hernández B., J.A., Izaguirre, H., Leon L., P., Lima, S., Mezones H., E., Monterrosa, A., Mostajo, D., Navarro, D., Ojeda, E., Onatra, W., Royer, M., Soto, E., Tserotas, K. y Vallejo, S. (2011). A large multinational study of vasomotor symptom prevalence, duration, and impact on quality of life in middle-aged women. Menopause, 18(7), 778-785. doi: 10.1097/ gme.0b013e318207851d.

Carrobles, A.M., Velasco, M. y Gómez, S. (2015). El concepto social de climaterio en población rural y urbana. European Journal of Investigation in Health, Psychology and Education, 5(2), 145-151.

Carvajal L., A., Flores R., M., Marín, S.I. y Morales, C.G. (2016). Los trastornos de ansiedad durante la transición a la menopausia. Perinatología y Reproducción Humana, 30(1), 39-45.

Charabati, D., Sanabria, V.H., y Sánchez, L. (2017). Intensidad de la sintomatología vasomotora y calidad de vida en mujeres obesas, en pacientes del Hospital Regional Tlalnepantla. Tesis de Especialidad. Toluca (México): Universidad del Estado de México.

Comino, D. y Gomiz, R. (1991). Epidemiología del climaterio. Revista de Medicina de la Universidad de Navarra, octubre-diciembre, 9-16.

Contreras G., Y.S. y González, A. (2015). Funcionamiento familiar en mujeres climatéricas sintomáticas usuarias del nivel primario de atención en Chile. Revista Cubana de Ginecología y Obstetricia, 41(4), 376-387.

Couto, D. y Nápoles, D. (2014). Aspectos sociopsicológicos del climaterio y la menopausia. Revista MEDISAN, 18(10), 1409-1418.

Dorador G., M.L. y Orozco C., G. (2018). Síntomas psicológicos en la transición menopáusica. Revista Chilena de Obstetricia y Ginecología, 83(3), 228-239.

Escobar C., F., Chica U., H.L. y Cuevas C., F. (2008). Trastornos del sueño relacionados con el climaterio femenino y su tratamiento. Revista Colombiana de Obstetricia y Ginecología, 59(2), 31-39.

García, M.C., Selva, A., Pérez, G.A. y Pardo, S. (2012). Eficacia de la terapia floral en trastornos de climaterio y menopausia. Revista Médica Multimed, 16(1). Recuperado de index.php/mtm/article/view/635.

Heras M., M., Recalde A., V., Rodríguez A., R., Bone L., I., Álava G., D. y Bermeo, L. (2017). Menopausia y actividad física: manual para la atención físico-educativa. Revista Cubana de Investigaciones Biomédicas, 36(2), 138-149.

Hernández V., M., Córdova P., N., Basurto, L., Saucedo, R., Vargas, C., Vargas, A., Ruiz, M., Manuel, L. y Zárate, A. (2010). Frecuencia de los síntomas del síndrome climatérico. Ginecología y Obstetricia de México, 78(4), 232-237.

Jaramillo M., M.C., Martínez. L., M. y Jaramillo L., I. (2016). Polimorfismos genéticos asociados a complicaciones crónicas de la menopausia. Revista de Obstetricia y Ginecología Venezolana, 76(1), 60-66.

Lauritzzen, C. (1984). EI climaterio de la mujer. Madrid: Raycar, S.A.

Legorreta, D., Montaño, J.A., Hernández, I., Salinas, C. y Hernández B., J.A. (2013). Edad en la menopausia, motivos de consulta y síntomas informados por mujeres mexicanas de 40 a 59 años de edad. Climacteric, 16(4), 417-425. doi: 10.3109 / 13697137.2012.696288.

Lopera V., J.S., Parada P., A.M., Martínez S., L.M., Jaramillo J., L.I. y Rojas J., S. (2016). Calidad de vida en la menopausia, un reto en la práctica clínica. Revista Cubana de Medicina General Integral, 32(3), 1-11.

Lugones, M. (2013). Climaterio, daño vascular y sexualidad. Revista Cubana de Obstetricia y Ginecología, 39(4), 413-426.

Martínez C., H.J.C. (2007). Menopausia y climaterio. Avances en ginecología: una mirada al futuro. Boletín Informativo de Laboratorios Janssen-Cilag, S.A. de C.V.

Martínez I., M., Sarduy, M., Rodríguez, L., Rodríguez, M. e Iglesias, B. (2016). Síntomas climatéricos según el estilo de vida en mujeres de edad mediana. Revista Cubana de Obstetricia y Ginecología, 40(3), 295-308.

Mercado L., M.F., Monterrosa C., A. y Duran M., L.C. (2018). Evaluación de la calidad de vida en climatéricas con la Escala Cervantes. Influencia de la etnia. Revista Peruana de Ginecología y Obstetricia, 64(1), 13-25. 
Morales, F., Díaz, E. y Aldana, E. (1995). Síntomas somáticos durante el climaterio asociados con estados depresivos. Perinatología y Reproducción Humana, 9(2), 85-92.

Pacheco R., J. (1994). Climaterio y menopausia. Ginecología y Obstetricia, 40(1), 6-23.

Pérez R., G.R., Martínez D., R., López B., M.T. y Ornat C., L. (2013). Síntomas relacionados con el climaterio en mujeres residentes en la provincia de Zaragoza. Enfermería Clínica, 23(6), 252-261.

Romero P.Z., I.M., Monterrosa C., A. y Paternina C., A. (2010). Menopausia etnias/razas: ¿Hay diferencia en la presentación de los síntomas? Revista Colombiana de Obstetricia y Ginecología, 61(4), 319-328.

Ulloque, L., Carriazo, S.M., Monterrosa, A. y Paternina, A. (2013). Climaterio: oleadas de calor y otros síntomas en indígenas zenúes colombianas. Revista de Investigaciones Andinas, 27(15), 744-758.

Utian, W. (1980). Menopause in modem perspective. New York: Appleton-Century-Crofts. 